\title{
Minimax Multivariate Control Chart Using a Polynomial Function
}

\author{
Johnson Ademola Adewara', Kayode Samuel Adekeye ${ }^{2}$, Osebekwin Ebenezer Asiribo ${ }^{3}$, \\ Samuel Babatope Adejuyigbe ${ }^{4}$ \\ ${ }^{1}$ Distance Learning Institute, University of Lagos, Akoka, Nigeria \\ ${ }^{2}$ Department of Mathematical Sciences, Redeemer's University, Redemption City, Nigeria \\ ${ }^{3}$ Department of Statistics, University of Agriculture, Abeokuta, Nigeria \\ ${ }^{4}$ Department of Mechanical Engineering, University of Agriculture, Abeokuta, Nigeria \\ E-mail: adewaraja@yahoo.com,samadek_2017@yahoo.co.uk,asiribo@yahoo.com \\ Received September 15, 2011; revised November 4, 2011; accepted November 12, 2011
}

\begin{abstract}
Minimax control chart uses the joint probability distribution of the maximum and minimum standardized sample means to obtain the control limits for monitoring purpose. However, the derivation of the joint probability distribution needed to obtain the minimax control limits is complex. In this paper the multivariate normal distribution is integrated numerically using Simpson's one third rule to obtain a non-linear polynomial (NLP) function. This NLP function is then substituted and solved numerically using Newton Raphson method to obtain the control limits for the minimax control chart. The approach helps to overcome the problem of obtaining the joint probability distribution needed for estimating the control limits of both the maximum and the minimum statistic for monitoring multivariate process.
\end{abstract}

Keywords: Minimax, Non-Linear Polynomial, Process, Maximum and Minimum

\section{Introduction}

Multivariate statistical process control (MSPC) is particularly important in the industries where data are collected on more than one variable. In practice, most of the quality characteristics to be controlled and monitored are not independent. The reason is that most of the variables involved are interconnected, that is, they are correlated. Hence, to monitor these interconnected or correlated variables is not simple but rather complex, especially for manufacturing processes. The use of multiple univariate control charts does not deliver a useful solution in this situation. The problems are that, the overall probability of signaling a false "out-of-control" situation is not controlled and more seriously the correlation among the variables are ignored.

In recent years, multivariate statistical process control (MSPC) procedures have enjoyed wide application in industry. This has resulted from expanded capability to monitor the key variables of a process with sensor and measurement technology, and the widespread availability of computers and statistical software programs that incorporate multivariate SPC capability.
Simultaneously, there have been many new technical developments that have made multivariate SPC more useful. For example, many authors have investigated methods of monitoring multivariate continuous data. [1] developed the multivariate $T^{2}$ statistic for quality control purposes. Multivariate generalizations of the CUSUM procedure have been studied by [2] and [3] developed and investigated multivariate exponentially weighted moving averages to identify quality problems. The use of multivariate exponentially weighted moving averages in monitoring multivariate data have been enhanced by [4] Monitoring principal components of multivariate data has been studied by [5]. [6] discussed multivariate minimax control chart and he used the joint probability distribution function of the minimum and maximum standardized sample means to derive the control limits to make decision if the process is in or out of control. However, the derivation of the joint probability distribution needed to obtain the minimax control limits as discussed by [6] is complex. In this paper we propose a Non-Linear Polynomial function (NLP) approach to multivariate minimax control chart to monitor continuous data as an alternative approach to the use of joint probability for both the ma- 
ximum $\left(Z_{[p]}\right)$ and minimum $\left(Z_{[1]}\right)$ limits used by [6]. The minimax control limits derived by [6] is modified and the multivariate normal distribution is integrated numerically using Simpson's one third rule to obtain a nonlinear polynomial (NLP) function. This NLP function is then substituted and solved numerically using Newton Raphson method to obtain the control limits for the minimax control chart.

\section{Non Linear Polynomial Function}

Polynomials are popular in curve and surface representations and many critical problems arising in Computer Aided Geometric Design such as surface integration, are reduced to finding the zero set of a system of nonlinear polynomial equations

$$
f(x)=0
$$

where $f=\left(f_{1}, f_{2} \cdots f_{n}\right)$ and each $f_{i}$ is a polynomial of independent variables $\underline{X}=\left(X_{1}, X_{2}, \cdots X_{l}\right)$. Several root-finding algorithms for multivariate polynomial systems (2.1) have been used in practice. Newton type methods, which are classified as local solution techniques, have been applied to many problems since they are quadratically convergent and produce accurate results. They, however, require good initial approximations of the roots of the systems, and fail to provide full assurance that all roots have been found. These limitations can be overcome by global solution technique, which can be categorized into three different types as proposed by [7]. The different types are algebraic and hybrid methods, homotropy methods, and subdivision methods. Among these techniques, the subdivision methods have been widely used in practice because of their performance and efficiency. The Interval Projected Polyhedral (IPP) algorithm proposed by [7] and [8] is one example, and it has been successfully applied to various problems. One particular interest is locating zeros of a univariate application of polynomial [9].

It is a critical problem in diverse fields such as control theory and many literature has been devoted to it (see e.g. [10]).

Most of the root finding algorithms, however, experience difficulties in dealing with roots with high multiplicity such as performance deterioration and lack of robustness in numerical computation. For example, the IPP algorithm, which belongs to the subdivision class of methods, slows down drastically and suffers from proliferation of boxes that are assumed to enclose roots. Moreover, since a root with high multiplicity is unstable with respect to small perturbation, round-off errors during floating point arithmetic may change the topological aspect in such a way that a cluster of roots could be formed around the root.
Solving univariate polynomials with multiple roots is an important but difficult task. [9] collated nine methods to bound multiple roots of polynomials and compared them rigorously. He also proposed a new hybrid algorithm which gives numerically nearly optimal bounds for multiple roots of univariate polynomials. Even though these methods work well in most cases, it is not easy for a user to control the size of the bound of a root in general. [11] used the Sturm sequences to compute all roots of a univariate polynomial, but his approach relies on the division of polynomials to compute Sturm sequences. So, it is not numerically robust unless exact arithmetic or symbolic computation is used.

This paper focuses on the particular case where the functions $f, i \quad \varepsilon\{1, \cdots, Q\}$ in $\left\{\begin{array}{l}x_{1}=f_{1}\left(s_{1}, \cdots, s_{N}\right) \\ \vdots=\quad \vdots \\ x_{Q}=f_{Q}\left(s_{1}, \cdots, s_{N}\right)\end{array}\right.$ are polynomials.

Thus, we write $X=f(s)$ where $f$ is a nonlinear function $f: C^{N} \rightarrow C^{Q}$ and $f_{1} \cdots f_{Q}$ constitute the component of $f$. The source separation problem consists of recovering the sources $s_{1} \cdots s_{N}$ from the observation $x_{1}, \cdots, x_{Q}$ for all $i, f_{i} \varepsilon C(s)$, where $C(s)$ stands for the set of polynomials in variables $s_{1}, \cdots, s_{N}$ and with coefficients in $C$. This restriction is partly justified by the difficulty to tackle the nonlinear case because of its generality. In addition, polynomials constitute an important class of nonlinear models which may represent acceptable approximations of certain nonlinearities.

Finally, an important reason to deal with this model is the following:

Consider the case where the multidimensional source vector belongs to a finite set $s \in \mathrm{A}=\left\{a^{(1)}, \cdots, a^{(n a)}\right\}$. Although seemingly restrictive, this situation is highly interesting since it occurs in digital communications, where the emitted source sequences belong to a finite alphabet depending on the modulation used.

An important observation is that if $s \varepsilon \mathrm{A}$ and $\mathrm{A}$ is finite, all instantaneous mixtures of the sources can be expressed as polynomial mixtures. This follows immediately from the fact that any function on a finite set can be interpolated by a polynomial in a way similar to Lagrange polynomial interpolation [12]. It follows that polynomial mixtures constitute the general model of nonlinear mixtures in the case of sources belonging to a finite alphabet.

\section{The Model}

$$
\left\{\begin{array}{l}
x_{1}=f_{1}\left(s_{1}, \cdots, s_{N}\right) \\
\vdots=\quad \vdots \\
x_{Q}=f_{Q}\left(s_{1}, \cdots, s_{N}\right)
\end{array}\right.
$$

is a polynomial, and in order to be able to resort to alge- 
braic techniques, we will restrict the separator to the class of polynomial functions in $x_{1}, \cdots, x_{Q}$, that is, $\forall_{i}, g_{i} \varepsilon C[x]$.

\subsection{Simpson One Third Rule}

The Simpson's $1 / 3^{\text {rd }}$ rule is a numerical method for finding the integral $\int_{a}^{b} f(x) \mathrm{d} x$ within some finite limits $a$ and $b$. Simpson's $1 / 3^{\text {rd }}$ rule approximates $f(x)$ with a polynomial of degree two $p(x)$, i.e., a parabola between the two limits $a$ and $b$, and then finds the integral of that bounded parabola, and is used to represent the approximate integral $\int_{a}^{b} f(x) \mathrm{d} x$. The integral of the approximated function is the area under the parabola bounded by the points $a$ and $b$ by the positive side of the $x$ axis. The quadratic function has three points common to the function $f(x)$, as follows: The end points of the approximate quadratic function $p(x)$ is the same as the function $f(x)$ at points $a$ and $b . p(x)$ takes the same value of the function $f(x)$ at point $m=(a+b) / 2$.

Thus three points are fixed each in equal interval $a>m>b$ and a parabola is drawn through these three points $f(a), f(m), f(b)$. The area under the parabola through these points bounded by $a$ and $b$ with the positive side of the $\mathrm{X}$ axis is found and used as the approximated integral value. The iterative formula below can be used to find the integral of a function $f(x)$ using Simpson's $1 / 3^{\text {rd }}$ rule.

$$
I_{S I}=\frac{h}{3}\left[f\left(x_{0}\right)+f\left(x_{1}\right)+f\left(x_{2}\right)\right]
$$

\subsection{Newton Raphson Method}

The Newton-Raphson method is based on the principle that if the initial guess of the root of $f(x)=0$ is at $x_{i}$, then if one draws the tangent to the curve at $f\left(x_{i}\right)$, the point $\left(x_{i+1}\right)$ where the tangent crosses the $x$-axis is an improved estimate of the root.

Using the definition of the slope of a function, at $x=x_{i}$

$$
f^{\prime}(x)=\tan \theta=\frac{f(x)-0}{x_{i}-x_{i+1}}
$$

Form Equation (2.4) we have

$$
x_{i+1}=x_{i}-\frac{f\left(x_{i}\right)}{f^{\prime}\left(x_{i}\right)}
$$

Equation (2.4) is called the Newton-Raphson formula for solving nonlinear equations of the form $f(x)=0$. So starting with an initial guess, $x_{i}$, one can find the next guess, $x_{i+1}$ by using Equation (2.5). One can repeat this process until one finds the root within a desir- able tolerance.

\section{Algorithm}

The steps of the Newton-Raphson method to find the root of an equation $f^{\prime}(x)=0$ are:

Step 1. Evaluate $f^{\prime}(x)$ symbolically

Step 2. Use an initial guess of the root, $i x_{i}$, to estimate the new value of the root, $x_{i+1}$ as $x_{i+1}=x_{i}-\frac{f\left(x_{i}\right)}{f^{\prime}\left(x_{i}\right)}$

Step 3. Find the absolute relative approximate error $\left|\varepsilon_{\alpha}\right|$ as

$$
\left|\varepsilon_{\alpha}\right|=\left|\frac{x_{i+1}-x_{i}}{x_{i+1}}\right| * 100
$$

Step 4. Compare the absolute relative approximate error with the pre-specified relative error tolerance, $\varepsilon_{s}$, If $\left|\varepsilon_{\alpha}\right|>\varepsilon_{\alpha}$, then go to Step 2, or else stop the algorithm. Also, check if the number of iterations has exceeded the maximum number of iterations allowed. If so, one needs to terminate the algorithm and notify the user.

\subsection{Minimax Control Chart}

The Minimax control chart developed by Sepulveda [6], and as discussed in [13] is similar to the charts proposed by [14] and [15]. The minimax control chart uses the minimum and maximum standardized sample means to make the decision if the process should be considered in control or out of control. However, the minimax chart uses both lower and upper control limits on both the maximum and minimum standardized sample means. This is facilitated by the development of the capability to determine the value of the joint density function of the maximum and minimum standardized sample means. This not only facilitates a method for setting the control limits, but also allows for the comparison of the performance of the minimax chart relative to other charts through computation of the out-of-control average run length.

Minimax control chart is used to standardize all $p$ means and to monitor the maximum and the minimum of those standardized sample means. To do this, the sample average vector $\underset{\sim}{X}=\left(\bar{X}_{1}, \bar{X}_{2}, \cdots, \overline{X_{p}}\right)$ is calculated and its elements are standardized using the expression:

$$
Z=\frac{\sqrt{n}(\bar{X}-\underline{\mu})}{\sigma}
$$

where $\mu$ is the population mean and $\sigma$ is the standard deviation. The vector $\underline{Z}=\left[Z_{i}\right], i=1,2, \cdots, p$ is now defined as the standardized sample mean vector. The maximum sample mean $\left(Z_{p}\right)$ is defined as the maximum of the elements of the vector $Z$, that is, $Z_{[p]}=\max \left(Z_{i}\right)$, Also, the minimum standardized sam- 
ple mean $\left(Z_{[1]}\right)$ is defined as the minimum of the elements of the vector $Z$.

The control limits that was proposed by [6] is modified to solve for both upper and lower of maximum and upper and lower of minimum as given in the expression below:

$$
\begin{aligned}
U C L_{[p]} & =\left\{\tau: \int_{-\infty}^{\tau} \cdots \int_{-\infty}^{\tau} f(Z) \mathrm{d} z=1-\alpha\right\} \\
L C L_{[p]} & =\left\{\tau: \int_{-\infty}^{\tau} \cdots \int_{-\infty}^{\tau} f(Z) \mathrm{d} z=\alpha\right\} \\
U C L_{[1]} & =\left\{\tau: 1-\int_{\tau}^{\infty} \cdots \int_{\tau}^{\infty} f(Z) \mathrm{d} z\right\} \\
L C L_{[1]} & =\left\{\tau: 1-\int_{\tau}^{\infty} \cdots \int_{\tau}^{\infty} f(Z) \mathrm{d} z=\alpha\right\}
\end{aligned}
$$

\section{Results}

The data for this research work were collected from the production line of a manufacturing company that produces soft drinks. The samples were drawn from the lines on each variable of the production. The data are secondary and multivariate in nature. The data had five variables which are: $X_{1}=$ Contents in ml, $X_{2}=$ Brev brix, $X_{3}$ = pressure, $X_{4}=$ Gas volume $\left(\mathrm{CO}_{2}\right)$ and $X_{5}=$ Temperature. Thus, $\underset{\sim}{X}=\left\{X_{1}, X_{2}, \cdots, X_{n}\right\}$. We assumed that the variables are normally distributed since we are dealing with continuous data. The multivariate normal distribution was integrated numerically using Simpson's one third rule. Simpson's rule is a numerical method that approximates the value of a definite integral by using quadratic polynomials. This approach was applied to the multivariate normal distribution to obtain a non-linear polynomial (NLP) function. This (NLP) function overcomes the problem of obtaining the joint probability distribution needed for the control limits of both the maximum $\left(Z_{[p]}\right)$ and the minimum $\left(Z_{[1]}\right)$ statistic. This method was used to determine the position of the five control limits of the chart stated in Equation (2.4). In other to obtain the control limits, an algorithm was developed and implemented on $C$ language to fit the polynomial function in the form $Z=0.0024 x^{5}+0.000005 x^{4}-$ $0.0444 x^{3}-0.00006 x^{2}+0.3805 x+0.4988$. Using the obtained polynomial equation, the algorithm in 2.2.1 was then used to obtain the control limits for both the minimum and the maximum statistics. The numerical solution for the control limits using the developed algorithm is presented in the Appendix. The control limits for minimum and maximum statistics for the five variables under consideration are presented in Table $\mathbf{1 .}$

Using the obtained control limits in Table 1, the process under study was tested for stability. To test for the stability of the process, Equation (2.6) was used to transform the data to obtain the minimum and the maximum
Table 1. The upper and lower control limit for both maximum and minimum statistics.

\begin{tabular}{cccc}
\hline$U C L_{[p]}$ & $L C L_{[p]}$ & $U C L_{[1]}$ & $L C L_{[1]}$ \\
\hline 2.4185 & 1.954 & 3.0306 & 2.7195 \\
-2.9458 & -1.3148 & -3.877 & -3.3942 \\
\hline
\end{tabular}

Table 2. The maximum and the minimum values.

\begin{tabular}{lllllll}
\hline Maximum & 0.018725 & 0.021426 & 0.021987 & 0.022794 & 0.0357959 \\
Minimum & 0.005012 & -0.00226 & -0.03127 & -0.03031 & -0.00954 \\
\hline
\end{tabular}

values for the five variables. The obtained minimum and maximum values are presented in Table 2.

\section{Discussion of Result}

The values in Table 2 are arranged from the lowest to the highest. Thus minimum of $Z_{[p]}$ is 0.018725 , and maximum of $Z_{[p]}$ is 0.0357959 . Also the minimum of $Z_{[1]}$ is -0.031271 and the maximum of $Z_{[1]}$ is 0.005012 . Comparing these values with the control limit in Table 1, the result shows that the minimum and maximum values obtained are within the control limits. Hence, the production process under consideration can be adjudged as being stable.

\section{Conclusions}

Minimax multivariate control chart is another sensitive multivariate control chart that has upper and lower control limits for both maximum and the minimum statistics for monitoring a multivariate process. The paper has addressed the use of numerical solution for obtaining the control limits of the minimax control chart as an alternative to the use of joint probability distribution.

\section{References}

[1] H. Hotelling, "Multivariable Quality Control_-Illustrated by the Air Testing of Sample Bombsights," In: C. Eisenhart, M. W. Hastay and W. A. Wallis, Eds., Techniques of Statistical Analysis, McGraw Hill, New York, 1947, pp. 111-184.

[2] W. H. Woodall and M. M. Ncube, "Multivariate Cusum Quality Control Procedures,” Technometrics, Vol. 27, No. 3, 1985, pp. 285-292. doi:10.2307/1269710

[3] C. A. Lowry, W. H. Woodall, C. W. Champ and S. E. Rigdon, "A Multivariate Exponentially Weighted Moving Average Control Chart,” Technometrics, Vol. 34, No. 1, 1992, p. 46. doi:10.2307/1269551

[4] G. C. Runger, J. B. Keats, D. C. Montgomery and R. D. Scranton, "Improving the Performance of the Multivariate Exponentially Weighted Moving Average Control Chart," Quality and Reliability International, Vol. 15, No. 
3, 1996, pp. 161-166.

doi:10.1002/(SICI)1099-1638(199905/06)15:3<161::AID -QRE215>3.0.CO;2-V

[5] C. M. Mastrangelo, G. C. Runger and D. C. Montgomery, "Statistical Process Monitoring with Principal Components," Quality and Reliability International, Vol. 12, No. 3, 1996, pp. 203-210. doi:10.1002/(SICI)1099-1638(199605)12:3<203::AID-Q RE12>3.0.CO;2-B

[6] A. Sepulveda, "The Minimax Control Chart for Multivariate Quality Control,” Dissertation, Department of Industrial and Systems Engineering, Virginia Polytechnic Institute and State University, Blacksburg, 1996.

[7] N. M. Patrikalakis and T. Maekawa, "Shape Interrogation for Computer Aided Design and Manufacturing," SpringerVerlag, Heidelberg, 2002.

[8] E.C. Sherbrooke and N. M. Patrikalakis, "Computation of the Solutions of Nonlinear Polynomial Systems," Computer Aided Geometric Design, Vol. 10, No. 5, 1993, pp. 379-405. doi:10.1016/0167-8396(93)90019-Y

[9] S. M. Rump, "Ten Methods To Bound Multiple Roots of Polynomials," Journal of Computational and Applied Mathematics, Vol. 156, No. 2, 2003, pp. 403-432. doi:10.1016/S0377-0427(03)00381-9
[10] .M. McNamee, “A Bibliography On Roots of Polynomials,” Journal of Computational and Applied Mathematics, Vol. 47, No. 3, 1993, pp. 391-394. doi:10.1016/0377-0427(93)90064-I

[11] H. S. Wilf, "A Global Bisection Algorithm for Computing the Zeros of Polynomials in the Complex Plane," Journal of the Association for Computing Machinery, Vol. 25, No. 3, 1978, pp. 415-420. doi:10.1145/322077.322084

[12] A. C. David, L. John and O. Donal, "Ideals, Varieties and Algorithms: An Introduction to Computational Algebraic Geometry and Commutative Algebra,” Springer, 2nd Edition, Berlin, 1996.

[13] J. Rehmert, "A Performance Analysis of the Minimax Multivariate Quality Control Chart,” M.Sc. Dissertation, Department of Industrial and Systems Engineering, Virginia Polytechnic Institute and State University, Blacksburg, 1997.

[14] A. J. Hayter and K. L. Tsui, "Identification and Quantification in Multivariate Quality Control Problems,” Journal of Quality Technology, Vol. 26, 1994, pp. 197-208.

[15] N. H. Timm, "Multivariate Quality Control Using Finite Intersection Tests,” Journal of Quality Technology, Vol. 28, 1996, pp. 233-243. 


\section{Appendix: Numerical Solution}

The numerical solutions for the control limits is

$$
Z(x)=0.0024 X^{5}+5 \times 10^{-5} X^{4}-0.444 X^{3}-6 \times 10^{-5} X^{2}+0.3805 X+0.4988
$$

The multiple integral of Equation (2.6) is solved below:

$$
\begin{aligned}
& \int\left(0.0024 X^{5}+5 \times 10^{-5} X^{4}-0.444 X^{3}-6 \times 10^{-5} X^{2}+0.3805 X+0.4988\right) \mathrm{d} x= \\
& \frac{0.0024 X^{6}}{6}+\frac{5 \times 10^{-6} X^{5}}{5}-\frac{0.444 X^{4}}{4}-\frac{6 \times 10^{-5} X^{3}}{3}+\frac{0.3805 X^{2}}{2}+0.4988 X \\
& \int\left(\frac{0.0024 X^{6}}{6}+\frac{5 \times 10^{-6} X^{5}}{5}-\frac{0.444 X^{4}}{4}-\frac{6 \times 10^{-5} X^{3}}{3}+\frac{0.3805 X^{2}}{2}+0.4988\right) \mathrm{d} x= \\
& \frac{0.0024 X^{7}}{7 \times 6}+\frac{5 \times 10^{-6} X^{6}}{6 \times 5}-\frac{0.444 X^{5}}{5 \times 4}-\frac{6 \times 10^{-5} X^{4}}{4 \times 3}+\frac{0.3805 X^{3}}{3 \times 2}+\frac{0.4988 X^{2}}{1 \times 2} \\
& \int\left(\frac{0.0024 X^{7}}{7 \times 6}+\frac{5 \times 10^{-6} X^{6}}{6 \times 5}-\frac{0.444 X^{5}}{5 \times 4}-\frac{6 \times 10^{-5} X^{4}}{4 \times 3}+\frac{0.3805 X^{3}}{3 \times 2}+\frac{0.4988 X^{2}}{1 \times 2}\right) \mathrm{d} x= \\
& \frac{0.0024 X^{8}}{7 \times 6 \times 8}+\frac{5 \times 10^{-6} X^{7}}{6 \times 5 \times 7}-\frac{0.444 X^{6}}{5 \times 4 \times 6}-\frac{6 \times 10^{-5} X^{5}}{4 \times 3 \times 5}+\frac{0.3805 X^{4}}{3 \times 2 \times 4}+\frac{0.4988 X^{3}}{1 \times 2 \times 3} \\
& \frac{0.0024 X^{9}}{7 \times 6 \times 8 \times 9}+\frac{5 \times 10^{-6} X^{8}}{6 \times 5 \times 7 \times 8}-\frac{0.444 X^{7}}{5 \times 4 \times 6 \times 7}-\frac{6 \times 10^{-5} X^{6}}{4 \times 3 \times 5 \times 6}+\frac{0.3805 X^{5}}{3 \times 2 \times 4 \times 5}+\frac{0.4988 X^{4}}{1 \times 2 \times 3 \times 4}
\end{aligned}
$$

The possible solutions for the numerical algorithm of the multiple integral are given below.

$$
\begin{aligned}
& -7.5249 \\
& -4.2219-4.8351 i \\
& -4.2219+4.8351 i \\
& -2.9458
\end{aligned}
$$

$\left.3.1708 \times 10^{3} x^{5}+2.0783 \times 10^{2} x^{4}-0.95\right)$, roots :

$0.24197-2.5085 i$

$0.24197+2.5085 i$

2.4185

$8.0042-4.6104 i$

$8.0042+4.6104 i$

$7.9366 \times 10^{-7} x^{9}+2.9763 \times 10^{-9} x^{8}-5.2857 \times 10^{-5} x^{7}-1.6667 \times 10^{-7} x^{6}+$

$$
\begin{aligned}
& -7.5651 \\
& -4.223-4.7726 i \\
& -4.223+4.7726 i \\
& -1.3148
\end{aligned}
$$

$\left.3.1708 \times 10^{-3} x^{5}+2.0783 \times 10^{-2} x^{4}-0.05\right)$, roots :

$$
\begin{aligned}
& 5.8498 \times 10^{-2}-1.2355 i, \text { roots } \\
& 5.8498 \times 10^{-2}+1.2355 i \\
& 1.1954 \\
& 8.0048-4.6049 i \\
& 8.0048+4.6049 i
\end{aligned}
$$




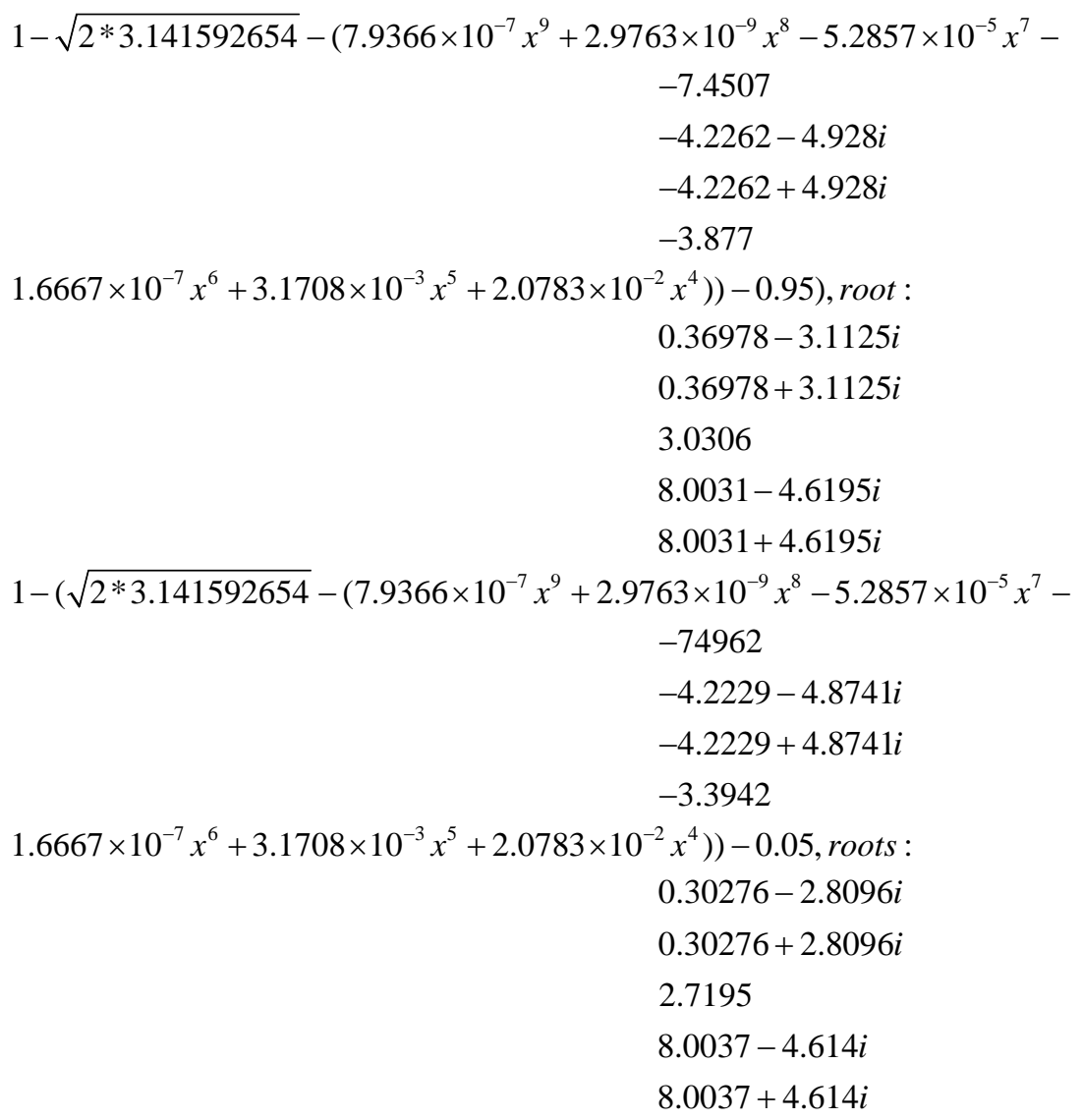

